\title{
Machine Learning Generalization of Lumped Parameter Models for the Optimal Cooling of Embedded Systems
}

\author{
Tudor George ALEXANDRU*, Cristina PUPĂZĂ \\ Politehnica University of Bucharest, 313 Splaiul Independenței, Bucharest 6, RO-060042, Romania \\ tudor.alexandru@upb.ro (*Correspondingauthor)
}

\begin{abstract}
Smart Retrofitting is an emerging approach that transforms conventional devices into Cyber-physical systems, thereby allowing enterprises to adhere to Industry 4.0. This paper proposes a thermal design methodology for Smart Retrofitting by means of Machine Learning that can be employed in order to generalize the thermal behaviour of embedded heat sources. The entire underlying heat transfer physics is captured by means of temperature dependent characteristics. The total heat transfer rate is estimated based on the software instruction cycle, allowing an accurate selection of the cooling components. This process is formalized by focusing on tangible aspects. Experimental studies are conducted on laboratory prototypes. The novelty of the study lies in the integration of Machine Learning for supporting Lumped Parameter simulations.
\end{abstract}

Keywords: Smart retrofitting, Embedded systems, Temperature sensing, Machine learning, Lumped parameter.

\section{Introduction}

Industry 4.0 represents an ongoing improvement of products and product related services for the development of interconnected manufacturing systems (Ghobakhloo, 2018). The industrial transformation of conventional computer control automation into smart devices is accomplished through a synergy between mechatronics, sensing and computational elements, supported by disruptive technologies, such as Cloud Computing and Internet of Things (Mehta et al., 2018), (Savu, Tomescu \& Băjenaru, 2017).

At the core of the Industry 4.0 platform stand Cyber Physical Systems (CPS) or intertwined embedded devices that exchange real-time data with the surrounding industrial ecosystem, which are characterized by an essential degree of intelligence and flexibility (Jazdi, 2014), (Dumitrache et al., 2017). Solving the complex system problems in such environments demands the wide-scale adoption of Machine Learning, as a fast and flexible way of predicting future events based on existing data (Stoica, 2019).

Smart Retrofitting can be defined as a set of methodologies, allowing enterprises to implement the concepts of Industry 4.0 by transforming conventional shop floor devices into Cyber Physical ones with the support of internal or external Embedded Systems (Guerreiro et al., 2018). The process is characterized by a wide range of workflows that relate to one another, in order to fully address the spatial and temporal scales peculiar to hardware and software problems (Beghi, Marcuzzi \& Rampazzo, 2016).
Among these workflows, the thermal design of Embedded Systems is an essential stage as hardware and software are concerned, when considering the limiting functional aspects involved and the ones related to performance (Krishna \& Koren, 2017). A wide body of literature has analysed the problem of adopting optimal cooling solutions that are applicable to Embedded Systems (Iranfar et al., 2017), (Cheng et al., 2017). Even so, the cross-linkage of hardware and software in Smart Retrofitting has increased the complexity of the thermal design process, which frequently leads to a demand for custom-tailored approaches.

In the past decades, the development of computer-aided software has supported the complex interdisciplinary thermal design with the help of virtual prototyping technologies (Hauck \& Jha, 2019). The simulation tools rely on experimental methods that can be deployed for performing both result verification on physical prototypes as well as data analytics for lowering the computational complexity in optimization processes (Marek, 2016), (Lin et al., 2018). Even so, the existing techniques demand extended knowledge of both simulation tools and experimental procedures, being less attractive for Smart Retrofitting projects.

The present work proposes a novel approach for the thermal design of Embedded Systems that combines supervised Machine Learning with Lumped Parameter simulations. At first, based on the specifications of the heat source subjected to thermal design, a range of electronic packages of similar configuration is decided 
upon. Experiments are conducted for capturing their thermal behaviour. The resulting knowledge is encompassed in a dataset side by side with datasheet specifications and performance metrics. Machine Learning is deployed in order to estimate heat transfer parameters corresponding to lumped conductance and capacitance. These two are used to parameterize the transient heat transfer analysis. Based on the loads imposed by the instruction cycles, the maximum temperature over time can be predicted, which allows an accurate optimal selection of cooling components.

Experimental procedures for contact and noncontact temperature measurements for embedded electronics are widespread throughout the literature (Gaofeng, Xueguang \& Qing, 2010). The use of Machine Learning for the timeseries prediction of the thermal behaviour of semiconductor chips based on Long shortterm memory and Gated recurrent units neural networks has recently been discussed (Yang \& Niu, 2019). Simplified numerical formulations, such as the lumped capacitance method have long been used for solving complex heat transfer problems with the help of computer programs (Benjamin et al., 1995). Most recent commercial heat transfer solvers implement such procedures in the form of user-definable macro elements (LMS Samtech, 2015).

Compared to the existing literature, the novelty of the aforementioned work consists in the development of a fully automated experimental procedure that is applicable to the study of embedded electronics, comprising multiple heat sources in the same case. The training and convergence issues peculiar to recurrent neural networks are tackled by deploying sequential models that predict steady-state heat transfer characteristics. A Lumped Parameter simulation is developed by using the Machine Learning logits and an imposed software resource usage.

This approach can successfully be deployed in Smart Retrofitting projects due to its low complexity and high reliance on tangible quantities. Furthermore, the ability of predicting the transient behaviour of embedded heat sources is a standpoint for other workflows, such as developing and tuning digital controllers (Wang, Yin \& Xiong, 2010) or optimizing cooling components for mass and power saving (Dede, Joshi \& Zhou, 2015).
The remainder of this paper is structured as follows: the limiting aspects of Embedded Systems thermal design are discussed in the context of Smart Retrofitting in the second section. The original contribution is highlighted in the third one by detailing each constitutive layer of the proposed approach with emphasis on the cross-linkage of the tools involved. A case study on the Smart Retrofitting of a computer control unit is included in the fourth section to prove the accuracy of this approach. Finally, the conclusion and a summary of the results are included in section five.

\section{Thermal Design in Smart Retrofitting}

Thermal design can be considered an informational subsystem development workflow that involves choosing the optimal cooling components for active heat sources, such as: microcontrollers, CPUs or networking chips. A holistic overview of the process is illustrated in Figure 1.

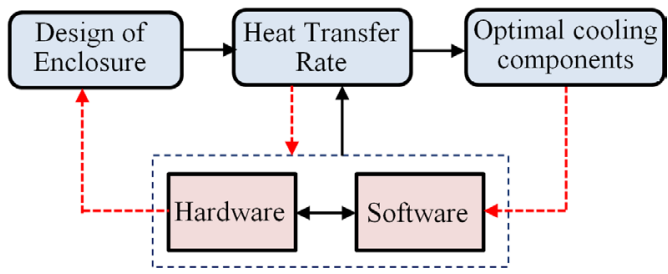

Figure 1. Holistic overview of the Thermal Design of Embedded Systems

In the first stage, the spatial scale of the enclosure setup is evaluated in terms of available space, allowable mass and operational power. The most critical aspect of thermal design is related to the second stage of the process, which involves the evaluation of the total heat transfer rate or the amount of power that is dissipated by the active heat sources during operation. Due to the fact that the software is written on top of the hardware, heat generation in embedded systems is a direct consequence of the involved computational elements combined with the resource scheduling algorithms. From this perspective, several engineering design loops can be distinguished, as frequent changes in hardware, software and enclosure layouts are systematically performed. For each derived configuration, an optimal selection of cooling components is completed, which results in a wide range of enclosure, 
hardware and/or software configurations. Based on technological, economical and enterpriserelated aspects, a final solution is adopted. When performing smart retrofitting, the flexibility of the thermal design process is severely diminished. This is due to the fact that most computercontrolled devices employed in the previous industrial transformation are not intended for future upgrades. From this perspective, only minor adjustments can be performed to the enclosures, while the software development is limited to the existing information exchange protocols.

The traditional approach for estimating the total heat transfer rate based on datasheet characteristics is no longer relevant due to its lack in capturing the heat transfer that is specific to each industrial environment. A study regarding the estimation of the total heat transfer rate for a CPU deployed in an embedded application was conducted, by comparing the datasheet Thermal Design Power (TDP) with experimentally derived values for the worst-case scenario of the operational conditions. The results presented in Table 1 indicate that the dissipated power is $61 \%$ lower than the one estimated by the manufacturer, which results in an excessive dimensioning of the heat sink or inadequate power requirements related to the cooling fan.

Table 1. Experimental assessment of the total heat transfer rate in an embedded $\mathrm{CPU}$

\begin{tabular}{|c|c|c|c|c|}
\hline Scenario & $\begin{array}{c}\mathbf{T}_{\mathbf{a}} \\
{\left[{ }^{\circ} \mathbf{C}\right]}\end{array}$ & $\begin{array}{c}\mathbf{R}_{\text {th }} \\
{\left[{ }^{\circ} \mathbf{C} / \mathbf{W}\right]}\end{array}$ & $\begin{array}{c}\mathbf{T}_{\text {max }} \\
{\left[{ }^{\circ} \mathbf{C}\right]}\end{array}$ & $\begin{array}{l}\text { Estimated } \\
\mathbf{T D P}[\mathbf{W}]\end{array}$ \\
\cline { 1 - 3 } Experiments & \multirow{2}{*}{25} & \multirow{2}{*}{4.36} & 67.9 & 9.85 \\
\cline { 1 - 1 } \cline { 1 - 1 } Datasheet & & & 95.2 & 16.12 \\
\hline
\end{tabular}

A strategy for adequately evaluating the total heat transfer rate in embedded systems is the multiphysics approach. By carrying out interdisciplinary analyses, the underlying heat source electromagnetics, the electric, fluid flow and heat transfer domains can be captured, which allows for a precise description of the thermal behaviour. Even so, the tools and resources involved in the process are appealing only for high-profile companies, as the cost and engineering knowledge demanded for SMEs in order to implement such procedures is excessively high. Nevertheless, the strategy proves a very low flexibility, as it over constrains the Smart Retrofitting process.
From these perspectives, a comprehensive approach for evaluating the total heat transfer rate in embedded systems is required by Thermal Design in Smart Retrofitting.

\section{Generalization of Lumped Parameter Models Based on Machine Learning}

In all embedded heat sources, the ability of semiconductors to oppose to the flow of energy releases heat at the package level and its surrounding junctions. At steady-state level, the phenomenon can be described by means of basic thermal circuits (Intel, 2017):

$R_{t h}=\frac{T_{\max }-T_{a}}{H_{f}}$

where: $\mathrm{R}_{\text {th }}$ is the thermal resistance $\left({ }^{\circ} \mathrm{C} / \mathrm{W}\right)$ occurring at the junction between two subcomponents, $\mathrm{T}_{\max }$ is the maximum temperature $\left({ }^{\circ} \mathrm{C}\right), \mathrm{T}_{\mathrm{a}}$ is the ambient temperature $\left({ }^{\circ} \mathrm{C}\right)$ and $\mathrm{H}_{\mathrm{f}}$ is the total heat transfer rate (W). Developing the equivalent thermal model of complex devices such as processors or Systems-on-a-Chip (SoC) can prove challenging due to the integrated circuit design density that causes complex power dissipation. The dominant heat flow in such components is characterized by the activity of the logic gates (Skadron, Stan \& Huang 2010):

$$
P_{\text {dynamic }}=C V^{2} \cdot f
$$

where: $\mathrm{P}_{\text {dynamic }}$ represents the released dynamic power (W), $C$ represents the package capacitance $(\mathrm{J} / \mathrm{K})$ and $\mathrm{f}$ the operating frequency $(\mathrm{MHz})$.

From equations 1 and 2, the thermal behaviour of any embedded heat source can be identified based on the ability of a package to conduct, store and release heat during software execution. Technical specifications and resource usage estimations can easily be derived based on product manuals and by evaluating the embedded software. Even so, every heat source has its own heat transfer peculiarities. Thus, developing a generalized model requires the identification of the relationship between the dissipated power and the temperature gradients in conjunction with the resource usage. For this purpose, the proposed approach is divided into three layers that correspond to the deployed software tools. A schematic representation of 


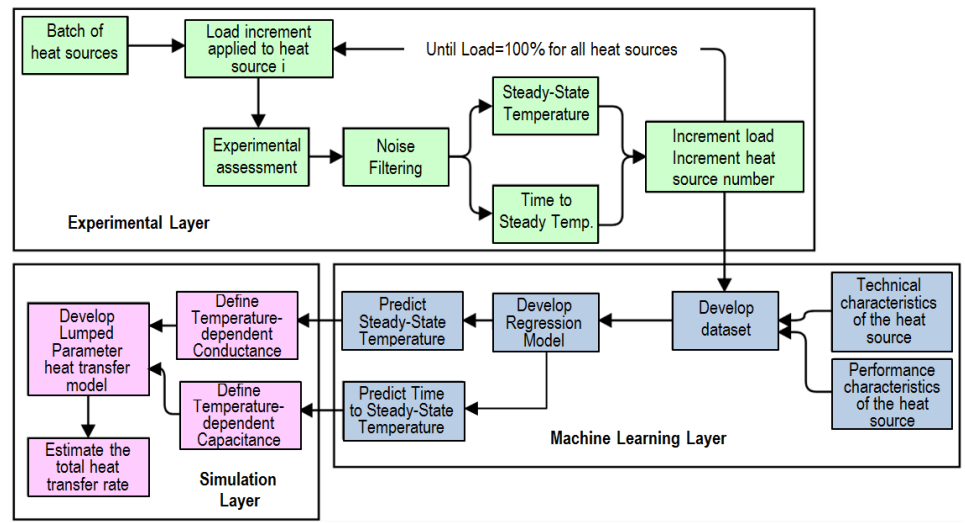

Figure 2. Schematic representation of the proposed approach

each layer is illustrated in Figure 2, followed by a brief description of each constitutive block in the following sections.

a) Experimental layer. An initial batch of heat sources is decided based on those already available at the level of shop floor computercontrolled devices (i.e. SoC, microcontrollers or CPUs). The aim of this study is to evaluate their behaviour when operating under constant instruction cycles. Various levels of resource usage percentages are considered, which allows one to carry out a wide range of measurements. The proposed experimental test platform comprises contact thermal sensors that are attached to the main junctions of the heat source. Alternatively, the task can be accomplished by means of internally embedded sensors. In this way the maximum temperature and time needed to achieve steady-state can be evaluated. These two characteristics provide a valuable insight in load-dependent conductance and capacitance. A software procedure for automating the process is depicted in Table 2.

Table 2. Experimental procedure

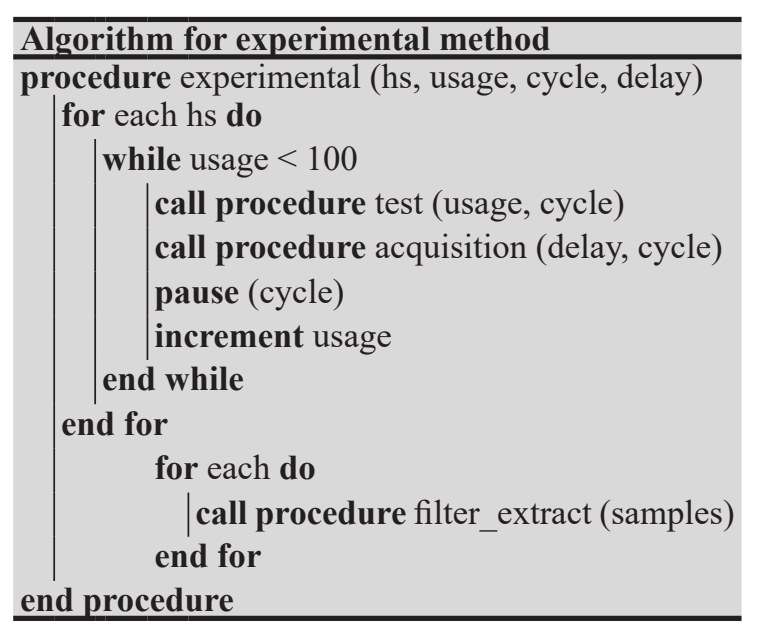

Embedded electronics such as multiprocessors require repeating the experimental procedure for each individual heat source and for every combination of heat sources (hs). An incremental resource usage is decided based on the resolution of the experimental system (usage). A standard cycle time (cycle) ensures that each acquisition takes place until steady-state is reached. The same variable can be employed in order to allow the device to cool down between measurements. On the other hand, a constant delay is applied to synchronize the execution of the procedures between the experimental and the test platform.

The main procedure calls other three ones. The instruction cycle is triggered on the test platform (test). This can be accomplished by monotonically executing the source code in low-complexity embedded systems. However, most recent devices used in CPS deploy operating systems, as they require various benchmarking procedures for adjusting resource usage and processor affinity. To remove any residual heat, all of the auxiliary functions of those devices must be turned off. At the same time, the temperature acquisition begins (acquisition), with a specified delay. For each completed measurement, a text file with the recorded time and temperature values (samples) is generated.

Regardless of the experimental method, the coexistence of perturbing factors, such as electromagnetic interference or variations in the ambient temperature results in noisy temperature acquisitions. From this perspective, a digital filter (i.e. Savitzky-Golay) is applied on the data points (filter_extract). All measurements are assembled in a single CSV file. Labels are added at the beginning and end of each experimental sequence for delimiting the acquisition cycles.

https://www.sic.ici.ro 
b) Machine Learning layer. The knowledge acquired in the experimental layer provides the necessary input for developing lumped models in the simulation layer. This allows one to estimate the transient behaviour of the heat sources based on their temperature-dependent heat transfer characteristics. Even so, this direct transfer does not provide any generalization ability. From this perspective, Machine Learning is added as an intermediate layer for supporting the knowledge development process. In this way, the model can adapt to new and unseen heat sources by training on existing experimental data.

A Deep Learning regression model is developed by means of a high level application programming interface. It comprises a dense neural network that can have several non-categorical inputs and one single continuous output, two hidden layers being defined (Figure 3).

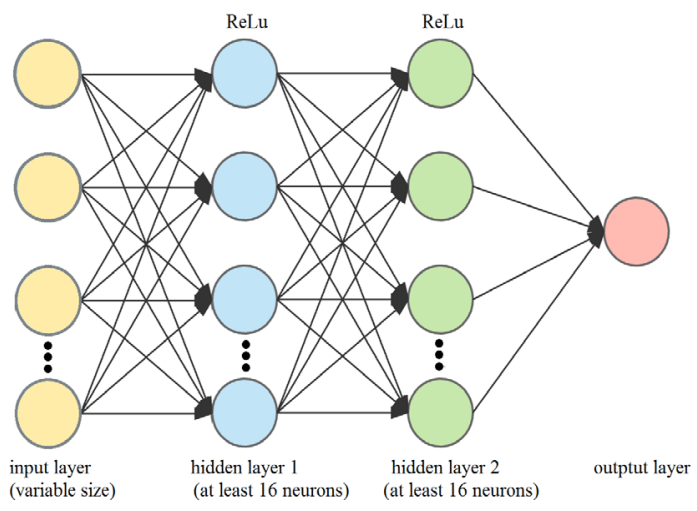

Figure 3. Deep Learning Sequential Neural Network with two hidden layers

The inputs applied to the neural network comprise the measurement sequences derived from the experimental layer. Additional labels are added to distinguish each heat source based on technical specifications that are in a close relationship with the power dissipation of the package. For example, a microcontroller chip requires a total of seven inputs. The first one is derived from the experimental layer, being a continuous value that describes the level of the resource usage. For this purpose, the test procedure can be carried out based on a variable resistance circuit applied to the microcontroller output pins, the obtained values resembling the resistance threshold. The other six features are extracted from the datasheet of the product, providing technical specifications that encompass relevant heat generation characteristics: manufacturer, program memory size, CPU Speed, SRAM, number of timers and operating voltage. An additional feature is added comprising scores of standardized benchmarks that can capture the computational efficiency of the device (i.e. Newton's Pi approximation). Onehot encoding is applied to the inputs to convert categorical features to numerical ones. Furthermore, min-max normalization is deployed to ease the training process.

In the output layer, the label corresponds either to the temperature of the heat sources embedded in the package or to the time required to achieve the steady-state temperature. The values are extracted from the measurements carried out in the experimental layer, being left unnormalized.

The solution that yielded the best results for a wide range of heat sources employed at least 16 neurons for the hidden layers. Rectified Linear Unit (ReLu) is used as activation function. Root Mean Square Propagation (RMSProp) optimization is deployed for adjusting the weights. A learning rate of 0.01 is defined in order to control the weights with respect to the gradient loss. Mean Squared and Mean absolute error loss functions are employed as model convergence metrics, 1000 Epochs being performed. These settings cause the training process to take a significant amount of time. Even so, it provides a fail-safe approach considering the small size of the datasets employed. Alternatively, if overfitting occurs, early stopping can be activated. Regardless of the adopted hyperparameters, the ability of the model to perform good predictions lies in the consistency of the dataset definition. A more complex example of labelled data used for multi-core CPUs is illustrated in Figure 4.

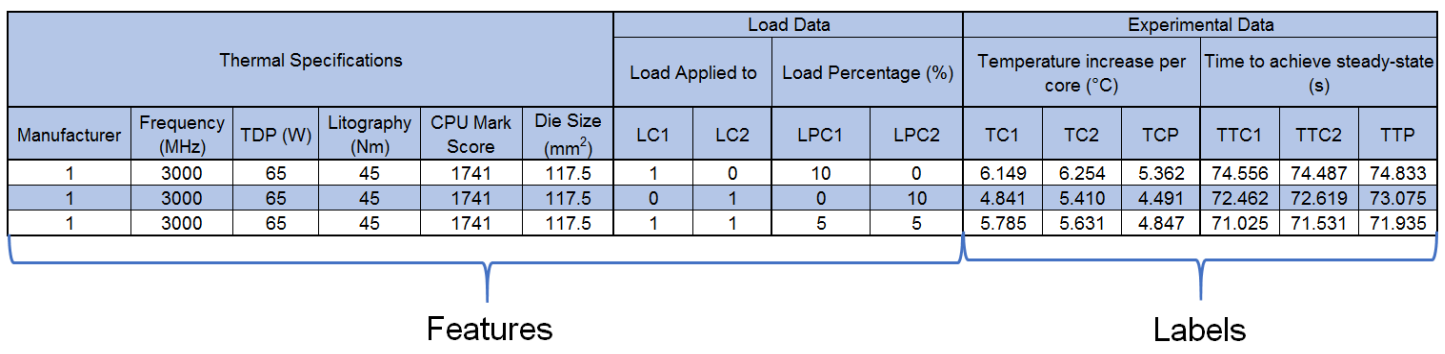

Figure 4. Example of a dataset used for multi-core CPUs with a selection of features and labels 
c) Simulation layer comprises a Finite Element Analysis (FEA) model. The computation of temperature distribution and heat flow adapted from (Zienkiewicz, Taylor \& Zhu, 2005) is described as:

$$
\rho c \frac{\partial T}{\partial t}=\partial_{i}\left(\lambda_{i j} \partial_{j} T\right)+Q
$$

where $\rho$ is the specific mass, $\mathrm{c}$ the specific heat, $\mathrm{T}$ the temperature, $\lambda$ the conductivity and $\mathrm{Q}$ the heat flux.

Instead of solving the complete heat transfer equations, the Lumped Parameter approach considers bodies without significant thermal gradients for nodal capacity, while 1D bars materialize the thermal interface conductance. 2D Skin elements are employed for modelling the package where each embedded heat source is formalized as a rectangular plate. Lead frame thermal resistance is modelled by means of coupled degrees of freedom (DOF). Contact elements are included so that the heat transfer path of the printed circuit board (PCB) is fully defined. The resulting macro element (Figure 5) requires as input the temperature-dependent conductance and capacitance, predicted in the Machine Learning layer. All other boundary conditions (i.e. surface convection) are considered constant.

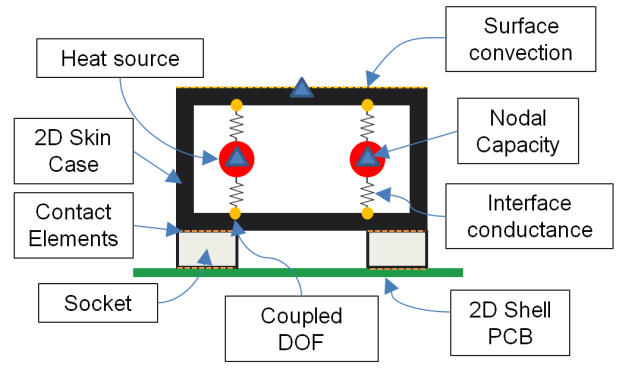

Figure 5. Representation of the macro element

\section{Evaluation of the Proposed Procedure for an Existing Computer Control Unit}

A case study on the Smart Retrofitting of an existing computer control unit is carried out. An internally deployed Embedded System including a dual core CPU is added to exchange real-time information with the existing hardware.

In this new setup, the processor is the main source of power dissipation, demanding a thermal design solution. The experimental setup is illustrated in Figure 6.

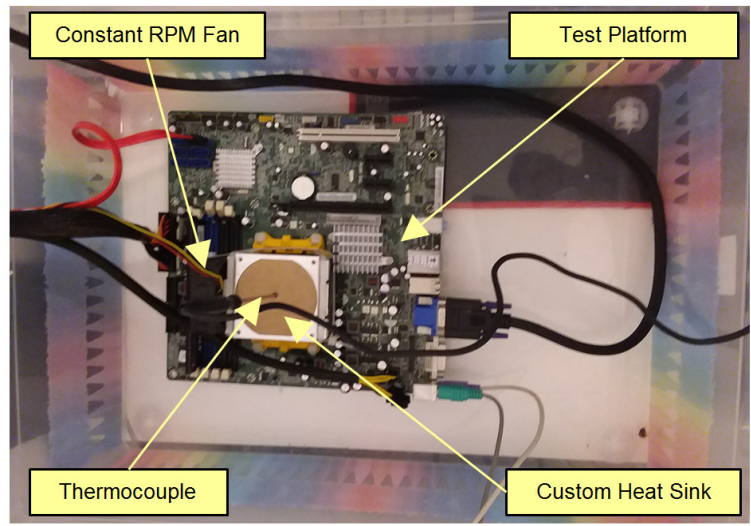

Figure 6. Experimental setup

The study is performed for a batch of 30 heat sources having a similar architecture to the embedded CPU, based on a selection of characteristics related to the thermal behaviour of the package involved, such as: manufacturer, operating frequency, size of the die and lithography technology. The resulting dataset comprises 10 feature columns: 6 technical and performance characteristics, 2 features specifying on which core the load is applied and 2 features which define the software usage. $80 \%$ of the dataset is considered for training the neural network, while the remaining $20 \%$ is used for validating its predictions.

The regression model is developed with the support of TensorFlow and Keras Deep Learning library.

The mean absolute error for 1000 Epochs in the case of the package temperature (TCP) is depicted in Figure 7 for the training set (Train Error) and the validation set (Val Error).

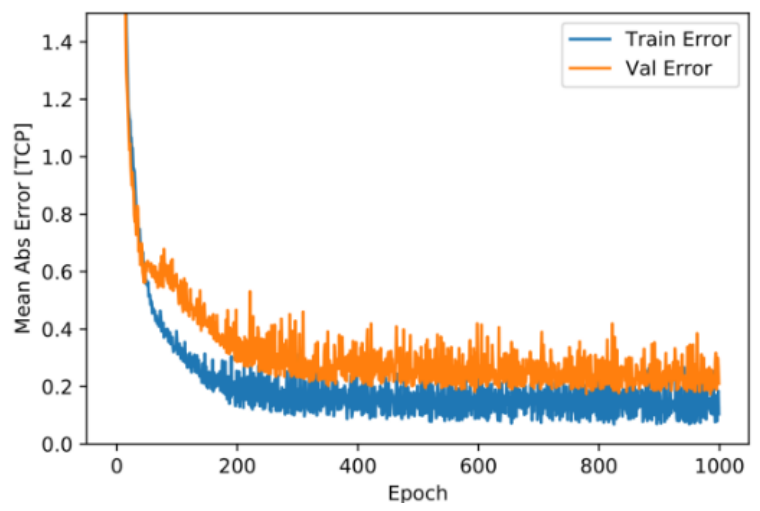

Figure 7. Learning curves for train set (Train Error) and validation set (Val Error) Mean Absolute Error

The graph indicates that the convergence of the model improves with the number of epochs. Furthermore, the trend of both curves is comparable, meaning that overfitting is avoided. 
In the validation set, the average prediction error is around $+/-0.4^{\circ} \mathrm{C}$ which provides a reasonable level of accuracy.

The Lumped Parameter model is completed using MSC Patran FEA software, while the solution is carried out using LMS SAMCEF Mecano Thermal solver (Figure 8).

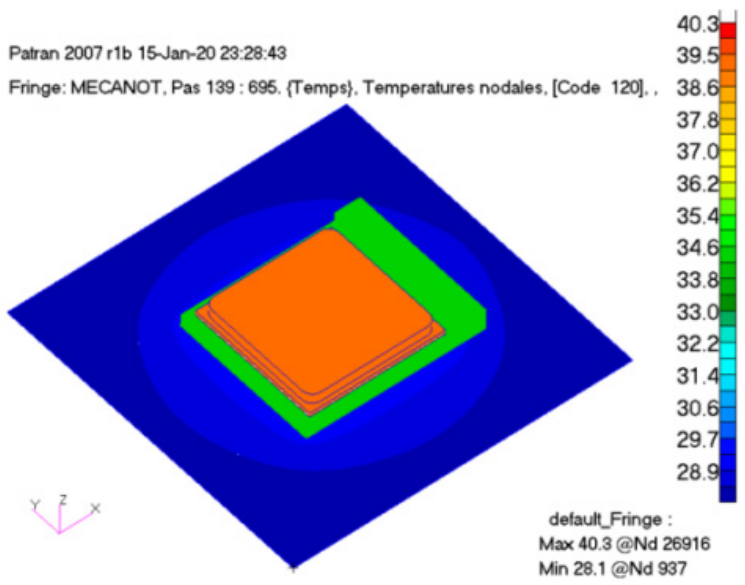

Figure 8. Fringe plot of the temperature distribution at steady-state

To test the overall accuracy of the approach, an embedded source code is executed to generate a transient resource usage on both cores of the CPU. An external thermocouple along with two sensors embedded in the die of the processor capture the temperature increase from the ambient at the main junctions.

An identical test cycle is considered in the Lumped parameter model, so that the resulting temperatures can be compared for both experiments and simulations considering the two cores and the package that embeds them (Figure 9).

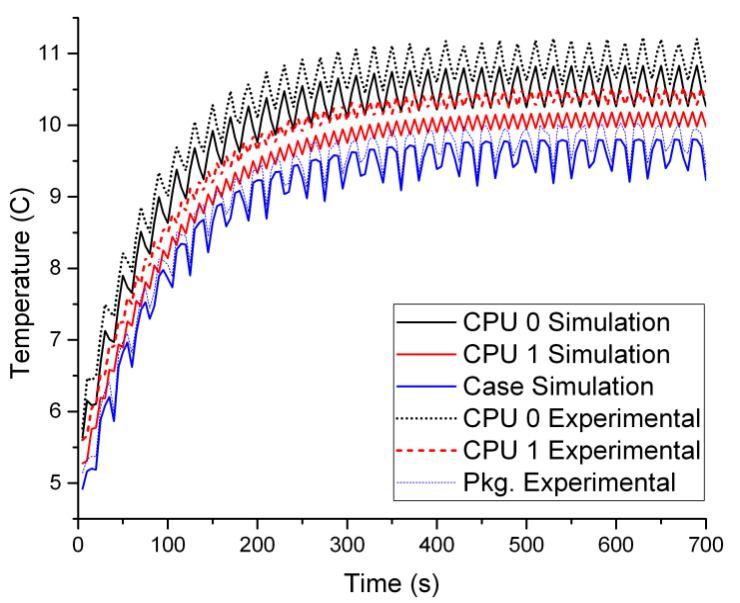

Figure 9. Temperature increase from the ambient for all cores (CPU 0 and 1) and the embedding package

$(\mathrm{Pkg})$ for both experiments and simulations
Experimental and simulation curves emphasize a similar temperature profile for all time steps, proving the ability of the proposed approach to approximate the conductance and capacitance of individual cores, as well as the package. In this regard, a mean absolute error of $0.34^{\circ} \mathrm{C}$ increase from the ambient was achieved for the first core, a $0.32^{\circ} \mathrm{C}$ increase from the ambient for the second one and a $0.17^{\circ} \mathrm{C}$ increase from the ambient for the package. The absolute error recorded at each time step for both experiments and simulation results is presented in Figure 10 as the average one achieved for all heat sources.

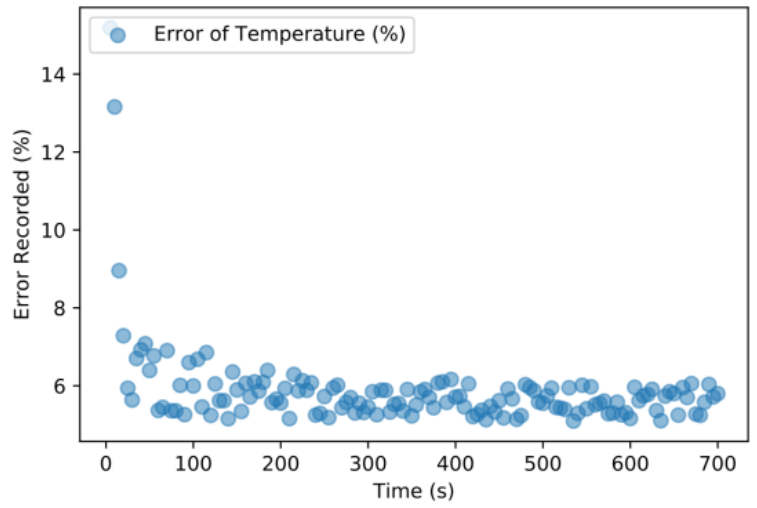

Figure 10. Average Error percentage related to the predicted temperature of the two cores and the package for each time step

The accuracy of the proposed model is low for the first recording intervals. This can be explained mainly by the limitations of the presented approach to capture thermal gradients when the heat sources are operating at low power. To overcome such issues, high-precision temperature acquisition methods can be deployed. Other sources of errors are related to the randomness peculiar to Machine Learning, as well as to the approximate methods deployed in the FEA.

Even so, the most important stage of the process is the evaluation of the total heat transfer rate. In this respect, a good match of the temperature increase from the ambient one can be noticed for both experimental and simulation attempts, proving good prediction accuracy.

\section{Conclusion}

This paper tackles the issues of Thermal Design in Smart Retrofitting by generalizing the behaviour of heat sources deployed in Embedded Systems with the support of Machine learning regression 
models. The proposed approach involves predicting heat transfer characteristics that resemble the conductance and capacitance of a package. These logits are further used in Lumped parameter simulation models. Best practices are presented throughout the work for easing the implementation process. A case study regarding the Smart Retrofitting of a computer control unit is provided to prove the accuracy of the presented approach. The results succeeded to capture the

\section{REFERENCES}

Beghi, A., Marcuzzi, F. \& Rampazzo, M. (2016). A Virtual Laboratory for the Prototyping of CyberPhysical Systems, IFAC-PapersOnLine, 49(6), 63-68.

Benjamin, A. S., Beraun, R., Brown, N. N. \& Sherman, M. P. (1995). Evaluation of conductive, radiative, chemical, and convective heat transfer in complex systems using a fast-running, implicit, lumped-capacitance formulation, No. SAND-950750C; CONF-950828-9. Sandia National Labs., Albuquerque, NM (United States).

Cheng, L., Zhao, Z., Huang, K., Chen, G. \& Knoll, A. (2017). McFTP: A framework to explore and prototype multi-core thermal managements on real processors. In $14^{\text {th }}$ IEEE International Conference on Embedded Software and Systems (ICESS 2017), (pp. 806-814).

Dede, E. M., Joshi, S. N. \& Zhou, F. (2015). Topology Optimization, Additive Layer Manufacturing, and Experimental Testing of an Air-Cooled Heat Sink, Journal of Mechanical Design, 137(11), 111403.

Dumitrache, I., Sacala, I. S., Moisescu, M. A. \& Caramihai, S. I. (2017). A conceptual framework for modeling and design of cyber-physical systems, Studies in Informatics and Control, 26(3), 325-334. DOI: $10.24846 / v 26 i 3 y 201708$

Gaofeng, H., Xueguang, Z. \& Qing, Y. (2010). Noncontact temperature measurement system based on embedded IIC. In 2010 International Conference on Computer Application and System Modeling (ICCASM 2010), 5 (pp. 43-46).

Ghobakhloo, M. (2018). The future of manufacturing industry: a strategic roadmap toward Industry 4.0, Journal of Manufacturing Technology Management, 26(6), 910-936.

Guerreiro, B. V., Lins, R. G., Sun, J. \& Schmitt, R. (2018). Definition of Smart Retrofitting: First steps for a company to deploy aspects of Industry 4.0, Advances in Manufacturing, 161-170.

Hauck, T. \& Jha, V. (2019). Virtual Prototyping and Simulation of Electro-Thermal Systems. In IEEE 20th transient temperature for the two cores of the CPU as well as the entire package with a maximum mean absolute error of $0.34{ }^{\circ} \mathrm{C}$ increase from the ambient. The approach is straightforward and can be further used for the evaluation of the total heat transfer rate based on the maximum temperatures over time. Thus, the selection of optimal cooling components can be performed by maintaining a subtle balance between the resources involved and the accuracy of the procedure.
International Conference on Thermal, Mechanical and Multi-Physics Simulation and Experiments in Microelectronics and Microsystems (EuroSimE), (pp. 1-4).

Intel (2017). Thermal management. Available at: $\quad<$ https://www.intel.com/content/www/us/en/ programmable/support/support-resources/operationand-testing/power/pow-thermal.html>.

Iranfar, A., Kamal, M., Afzali-Kusha, A., Pedram, M. \& Atienza, D. (2017). TheSPoT: Thermal StressAware Power and Temperature Management for Multiprocessor Systems-on-Chip, IEEE Transactions on Computer-Aided Design of Integrated Circuits and Systems, 37(8), 1532-1545.

Jazdi, N. (2014). Cyber physical systems in the context of Industry 4.0. In 2014 IEEE International Conference on Automation, Quality and Testing, Robotics (pp. 1-4).

Krishna, C. M. \& Koren, I. (2017). Thermal-aware management techniques for cyber-physical systems, Sustainable Computing: Informatics and Systems, 15, 39-51.

Lin, Q., Hong, J., Liu, Z., Li, B. \& Wang, J. (2018). Investigation into the topology optimization for conductive heat transfer based on deep learning approach, International Communications in Heat and Mass Transfer, 97, 103-109.
LMS Samtech
(2015).
Samcef
V15.1
User's Manual.

Marek, V. (2016). Basic research of thermal transfer simulations, Proceedings in Manufacturing Systems, 11(3), 119-125.

Mehta, P., Rao, P., Wu, Z. D., Jovanović, V., Wodo, O. \& Kuttolamadom, M. (2018). Smart Manufacturing: State-of-the-Art Review in Context of Conventional and Modern Manufacturing Process Modeling, Monitoring and Control. In Proceedings of the ASME 2018 13th International Manufacturing Science and Engineering Conference MSEC2018, 3(1), (pp.1-21). 
Savu, D., Tomescu, M. \& Băjenaru, L. (2017). Internetul Lucrurilor - $\mathrm{O}$ nouă paradigmă a conectarii în Internet, Revista Română de Informatică și Automatică, 27(1), 5-14.

Skadron, K., Stan, M. \& Huang, W. (2010). Thermal Modeling for Processors and Systems-on-Chip, Processor and System-on-Chip Simulation, 243-257.

Stoica, I. (2019). Solving System Problems with Machine Learning, Studies in Informatics and Control, 28(2), 119-132. DOI: 10.24846/v28i2y201901

Wang, Z., Yin, Z. \& Xiong, Y. (2010). Temperature Control and PID Parameters Optimization Based on Finite Element Model. In 2010 International Conference on Electrical and Control Engineering (pp. 2241-2244).

Yang, X. \& Niu, Q. (2019). Comparison of Longshort Term Memory against Gate Recurrent Unit on Semiconductor Die Temperature Fitting. In 2019 IEEE $2^{\text {nd }}$ International Conference on Automation, Electronics and Electrical Engineering (AUTEEE) (pp. 256-260).

Zienkiewicz, O. C., Taylor, R. L. \& Zhu, J. Z. (2005). The Finite Element Method: Its Basis and Fundamentals. Elsevier. 